\title{
Noise-induced temporal dynamics in Turing systems
}

\author{
Linus J. Schumacher \\ Centre for Mathematical Biology, Mathematical Institute, University of Oxford, 24-29 St. Giles', Oxford, OXI 3LB, United Kingdom and \\ Computational Biology Group, Department of Computer Science, University of Oxford, Oxford OXI 3QD, United Kingdom \\ Thomas E. Woolley \\ Oxford Centre for Collaborative Applied Mathematics, Mathematical Institute, University of Oxford, 24-29 St. Giles', \\ Oxford, OXI 3LB, United Kingdom \\ Ruth E. Baker \\ Centre for Mathematical Biology, Mathematical Institute, University of Oxford, 24-29 St. Giles', Oxford, OX1 3LB, United Kingdom
}

(Received 5 March 2013; published 25 April 2013)

\begin{abstract}
We examine the ability of intrinsic noise to produce complex temporal dynamics in Turing pattern formation systems, with particular emphasis on the Schnakenberg kinetics. Using power spectral methods, we characterize the behavior of the system using stochastic simulations at a wide range of points in parameter space and compare with analytical approximations. Specifically, we investigate whether polarity switching of stochastic patterns occurs at a defined frequency. We find that it can do so in individual realizations of a stochastic simulation, but that the frequency is not defined consistently across realizations in our samples of parameter space. Further, we examine the effect of noise on deterministically predicted traveling waves and find them increased in amplitude and decreased in speed.
\end{abstract}

DOI: 10.1103/PhysRevE.87.042719

PACS number(s): 87.18.Hf, 82.40.Ck, 82.20.Fd, 87.18.Tt

\section{INTRODUCTION}

Pattern formation constitutes a central puzzle of developmental biology and morphogenesis. It addresses, among other things, the fundamental question of how the complex structure of an organism arises from a single zygote and how it does so consistently despite varying environmental influences and the inherent stochasticity that arises from biochemical reactions involving small copy numbers.

Many mathematical models of pattern formation suffer from the fine-tuning problem: The conditions for pattern formation usually put strong restrictions on the allowed parameter space and/or the initial conditions [1]. Further, the precise morphology of the patterns generally has a strong parameter dependence. This might provide a mechanism of generating biological variation, but suggests that pattern formation only occurs in a small region of parameter space, which runs contrary to our demand that mechanistic models be robust in the face of noise.

One popular framework for describing pattern formation uses reaction-diffusion equations (RDEs) to produce the necessary symmetry breaking. Typically, such pattern-forming RDE systems involve two or more species interacting via short-range activation and long-range inhibition kinetics that tend to a homogeneous stable steady state in the absence of diffusion. With the addition of diffusion and a large enough domain, this steady state can be driven unstable, leading to spatial heterogeneity. As such, these models are said to undergo a diffusion-driven or Turing instability. This mechanism was first postulated in 1952 [2]. Although Turing systems do suffer from the fine-tuning problem, over the past decade it has been found that this can be alleviated, counterintuitively, by the inclusion of intrinsic noise through a stochastic modeling framework [1].

Even though Turing patterns are sensitive to perturbations and fine-tuning of their parameters, intrinsic noise can have a constructive effect on them insofar as it increases the parameter region in which patterns form [3-6]. Moreover, a number of models have been shown to exhibit other noise-induced phenomena such as quasiperiodic and chaotic oscillations [7], quasicycles [8], enhanced amplitude of oscillations [9], waves [10], and metastability [11]. These noise-induced phenomena have mainly been detected by spectral methods $[4,5,8,9,12]$, but other measures of order parameter statistics have been suggested [13]. The focus has largely been on intrinsic noise, but extrinsic noise has, in some cases, been observed to have qualitatively similar effects [4].

In this paper we apply a combination of analytical and numerical techniques to further understand the role of noise in pattern formation, in particular with respect to temporal behaviors such as polarity switching. In Sec. II we introduce the reaction system studied in this work and derive analytic results under a linear noise approximation. We compare these with stochastic simulations in Sec. III, exploring parameter space in a search for noise-induced spatiotemporal behaviors. In particular, we inspect the behavior near bifurcation boundaries of the deterministic system and classify the behavior of the stochastic system by identifying characteristic peaks in the power spectrum. In addition, we examine the change of deterministically predicted spatiotemporal dynamics when noise is included, i.e., the effect of intrinsic noise on oscillating patterns or traveling waves. We conclude by discussing our results in Sec. IV.

\section{A. Polarity switching}

A particular spatiotemporal behavior that can be observed in stochastic reaction-diffusion systems, even when the deterministic system has a stable steady state, is polarity switching. By this we mean when, in a pattern that has formed, peaks switch to troughs and vice versa, so that the pattern remains, but with the opposite polarity. In one spatial dimension this 


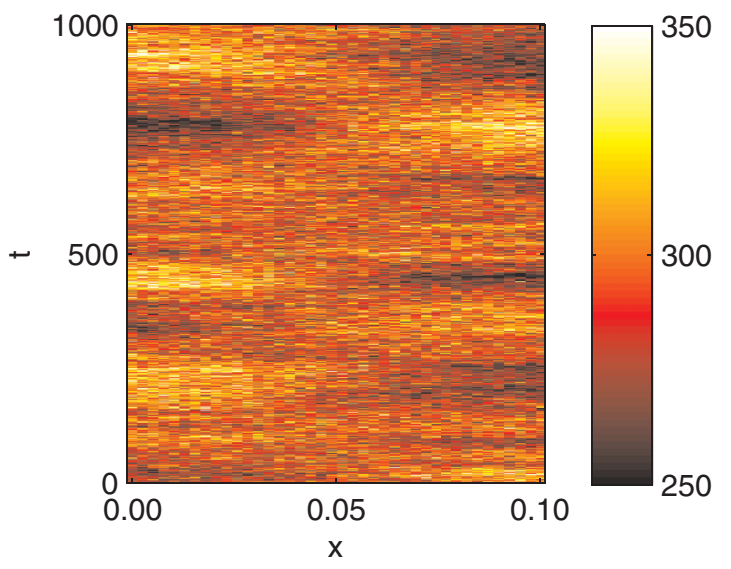

FIG. 1. (Color online) Realization of a stochastic simulation of the Schnakenberg reaction system on a one-dimensional domain, showing repeated polarity switching over time. Only the activator dynamics are shown and the range has been limited for visual emphasis.

corresponds to a degeneracy between patterns of the form $+\cos (k x)$ and those of the form $-\cos (k x)$, for example. We use different terms to distinguish between polarity switching, which is observed only in the stochastic system, and oscillating patterns, which are predicted deterministically. Still, polarity switching can occur in a seemingly oscillatory fashion, where a pattern oscillates back and forth between opposite polarities (see Fig. 1). It is hitherto unknown whether there is a defined frequency, or range of frequencies, for this polarity switching and how this might depend on the reaction parameters. This is one of the main questions we set out to investigate with this work and along the way we chart the range of possible spatiotemporal dynamics for the particular reaction system analyzed.

\section{METHODS}

We adopt a lattice-based stochastic approach to study our reaction-diffusion system. In particular, we study the behavior of a system of particles on a one-dimensional domain of length $L$, discretized into $K$ compartments. Particles can react with other particles in the same compartment or they can diffuse into directly adjacent compartments. We describe this system mathematically with a reaction-diffusion master equation
(RDME). Using a weak-noise, or system size, expansion [14], we conduct an analytic study of the behavior of fluctuations around the steady state, which are of order $\sqrt{\Omega}$, where $\Omega$ is the system size. This analysis can be equivalently described by a statistical field theory formalism $[3,4]$. However, since the results are the same, we stick with the majority of the field and use matrix methods developed by Elf and Ehrenberg [15]. This formalism has been developed extensively. Thus we highlight the key points here and refer the interested reader to work by Woolley et al. [12] (and references therein) or Lugo and McKane [5] for derivations of the RDME, weak-noise expansion, and Fokker-Planck equation. The details of this approach and the particular reaction kinetics are now outlined.

\section{A. Schnakenberg kinetics}

As an example of a reaction-diffusion system with a Turing instability we choose the Schnakenberg (or Gierer-Meinhardt) kinetics [16,17]. Even though we focus on this particular reaction system, the analysis applies to any set of kinetics mutatis mutandis. The Schnakenberg reactions are

$$
\emptyset \underset{\kappa_{-1}}{\stackrel{\kappa_{1}}{\rightleftharpoons}} U, \quad \emptyset \stackrel{\kappa_{2}}{\rightarrow} V, \quad 2 U+V \stackrel{\kappa_{3}}{\rightarrow} 3 U .
$$

Particle numbers of the two species are denoted by $U$ and $V$, respectively, whereas substances not explicitly modeled are denoted by $\emptyset$. The $\kappa_{i}$ specify the (stochastic) reaction rates.

\section{B. Reaction-diffusion master equation}

To model a reaction-diffusion system stochastically in a spatially extended, discretized domain, we start from the RDME. This approach models diffusion as reactions between compartments, with rates equal to the diffusion constants scaled according to the level of discretization. For a onedimensional domain of length $L$ discretized into $K$ compartments, the reaction rates for diffusion are $d_{U}=D_{u} /\left(\Delta_{x}\right)^{2}$ and $d_{V}=D_{v} /\left(\Delta_{x}\right)^{2}$, where $D_{u}$ and $D_{v}$ are the diffusion constants of the chemical species $U$ and $V$, respectively, and $\Delta_{x}=L / K$ is the length of a single compartment. We impose no-flux boundary conditions at each end of the domain. The RDME describes the time evolution of the probability $P\left(\mathbf{U}, \mathbf{V}, t \mid \mathbf{U}_{0}, \mathbf{V}_{0}, t_{0}\right)$ that the system is in a particular state at time $t$, defined by the particle numbers in each compartment, $\mathbf{U}=\left(U_{1}, \ldots, U_{K}\right)$ and $\mathbf{V}=\left(V_{1}, \ldots, V_{K}\right)$, conditional on the particle distributions $\mathbf{U}_{0}$ and $\mathbf{V}_{0}$ at time $t_{0}$. The RDME for the one-dimensional Schnakenberg system can be written as

$$
\begin{aligned}
\frac{\partial P}{\partial t}= & \sum_{i=1}^{K-1} d_{U}\left[\left(U_{i}+1\right) P\left(\ldots, U_{i}+1, U_{i+1}-1, \ldots\right)-U_{i} P\right]+\sum_{i=2}^{K} d_{U}\left[\left(U_{i}+1\right) P\left(\ldots, U_{i-1}-1, U_{i}+1, \ldots\right)-U_{i} P\right] \\
& +\sum_{i=1}^{K-1} d_{V}\left[\left(V_{i}+1\right) P\left(\ldots, V_{i}+1, V_{i+1}-1, \ldots\right)-V_{i} P\right]+\sum_{i=2}^{K} d_{V}\left[\left(V_{i}+1\right) P\left(\ldots, V_{i-1}-1, V_{i}+1, \ldots\right)-V_{i} P\right] \\
& +\sum_{i=1}^{K}\left\{\kappa_{1}\left[P\left(\ldots, U_{i}-1, \ldots\right)-P\right]+\kappa_{-1}\left[\left(U_{i}-1\right) P\left(\ldots, U_{i}+1, \ldots\right)-U_{i} P\right]+\kappa_{2}\left[P\left(\ldots, V_{i}-1, \ldots\right)-P\right]\right. \\
& \left.+\kappa_{3}\left[\left(U_{i}-1\right)\left(U_{i}-2\right)\left(V_{i}+1\right) P\left(\ldots, U_{i}-1, \ldots, V_{i}+1, \ldots\right)-U_{i}\left(U_{i}-1\right) V_{i} P\right]\right\} .
\end{aligned}
$$


For readability we have used a shorthand for the probabilities, omitting the unchanging variables as well as the conditionality on initial values. The first two sums on the right-hand side of (2) describe the diffusion of species $U$ to the right and left, respectively, the third and fourth sums accordingly describe the diffusion of $V$. The last sum represents the Schnakenberg kinetics, as stated in (1).

\section{System size expansion}

Since we are interested in understanding the noise-induced spatiotemporal dynamics of our system, we follow the formalism of expanding our population variables in the system size parameter $\Omega$,

$$
U_{i}=u_{i} \Omega+\eta_{u_{i}} \sqrt{\Omega}, \quad V_{i}=v_{i} \Omega+\eta_{v_{i}} \sqrt{\Omega} .
$$

A consistent choice for the system size parameter $\Omega$ can be made as the smaller of the two steady state populations, i.e., $\Omega=\min \left(U_{s}, V_{s}\right)$ [12], where $U_{s}$ and $V_{s}$ will be derived in the deterministic limit (see Sec. II C1).

\section{Deterministic limit}

From here we can carry out the corresponding expansion of the RDME (2) and collect terms of matching order in $\Omega$. The leading-order terms reproduce the deterministic behavior:

$$
\begin{aligned}
& \frac{\partial u}{\partial t}=D_{u} \nabla^{2} u+c_{1}-c_{-1} u+c_{3} u^{2} v, \\
& \frac{\partial v}{\partial t}=D_{v} \nabla^{2} v+c_{2}-c_{3} u^{2} v
\end{aligned}
$$

where $u$ and $v$ are the particle concentrations, $D_{u}$ and $D_{v}$ the diffusion constants, and the $c_{i}$ the deterministic reactions rates. The concentrations are related to the particle numbers through the system size $\Omega$ by $u=U / \Omega$ and $v=V / \Omega$ and the deterministic reaction rates are related to the stochastic reaction rates through the scaling relations $c_{1,2}=\kappa_{1,2} / \Omega$ and $c_{-1}=\kappa_{-1}, c_{3}=\kappa_{3} \Omega^{2}$. The deterministic limit gives us the steady states $U_{s}=\left(\kappa_{1}+\kappa_{2}\right) / \kappa_{-1}$ and $V_{s}=\kappa_{2} / \kappa_{3} U_{s}^{2}$, which will in turn determine our choice of $\Omega=\min \left(U_{s}, V_{s}\right)$.

\section{Langevin dynamics}

The next lowest order of the system size expansion of the RDME results in a Fokker-Planck equation for the dynamics of the probability density $[12,18]$. Alternatively, Gillespie has shown how "the microphysical premise from which the chemical master equation is derived leads directly to an approximate time-evolution equation of the Langevin type" [19]. We will proceed by assuming that the explicit dynamical conditions for this approximation to be accurate are fulfilled and simply quote the result without deriving the Fokker-Planck equation first.

The Langevin equation for a two-species reaction-diffusion system and its noise covariances are given by [12]

$$
\begin{aligned}
\frac{\mathrm{d} \zeta(t)}{\mathrm{d} t} & =\mathbf{A} \zeta(t)+\lambda(t), \\
\left\langle\lambda_{i}(t) \lambda_{j}\left(t^{\prime}\right)\right\rangle & =B_{i j} \delta\left(t-t^{\prime}\right),
\end{aligned}
$$

where $\zeta=\left(\eta_{u_{1}}, \ldots, \eta_{u_{K}}, \eta_{v_{1}}, \ldots, \eta_{v_{K}}\right)^{\mathrm{T}}$ denotes the fluctuations in species population numbers and in our case the matrices are $2 K \times 2 K$ and have the forms

$$
\mathbf{A}=\left(\begin{array}{ll}
\mathbf{a} & \mathbf{b} \\
\mathbf{c} & \mathbf{d}
\end{array}\right), \quad \mathbf{B}=\left(\begin{array}{ll}
\boldsymbol{\alpha} & \boldsymbol{\beta} \\
\boldsymbol{\beta} & \boldsymbol{\gamma}
\end{array}\right) .
$$

All submatrices are symmetric; $\mathbf{a}, \mathbf{d}, \boldsymbol{\alpha}$, and $\boldsymbol{\gamma}$ are tridiagonal; and $\mathbf{b}, \mathbf{c}$, and $\boldsymbol{\beta}$ are diagonal. Denoting diagonal entries of a matrix $\mathbf{M}$ with $m_{0}$ and sub- and superdiagonal entries with $m_{1}$, the reaction rate matrices for the Schnakenberg system are as follows:

$a_{0}=-2 d_{U}-\kappa_{-1}+2 u_{s} v_{s} \kappa_{3} \Omega^{2}, \quad a_{1}=d_{U}$,

$b_{0}=u_{s}^{2} \kappa_{3} \Omega^{2}, \quad c_{0}=-2 u_{s} v_{s} \kappa_{3} \Omega^{2}, \quad d_{0}=-2 d_{V}-u_{s}^{2} \kappa_{3} \Omega^{2}$,

$d_{1}=d_{V}, \quad \alpha_{0}=4 d_{U} u_{s}+\frac{\kappa_{1}}{\Omega}+\kappa_{-1} u_{s}+u_{s}^{2} v_{s} \kappa_{3} \Omega^{2}$,

$\alpha_{1}=-2 d_{U} u_{s}, \quad \beta_{0}=-u_{s}^{2} v_{s} \kappa_{3} \Omega^{2}$,

$\gamma_{0}=4 d_{V} v_{s}+\frac{\kappa_{2}}{\Omega}+u_{s}^{2} v_{s} \kappa_{3} \Omega^{2}, \quad \gamma_{1}=-2 d_{V} v_{s}$.

\section{Temporal Fourier spectrum}

Since we want to study patterning in the presence of dynamic behavior, we are ultimately interested in the spatiotemporal power spectrum, but let us first derive just the temporal spectrum to recapitulate the methods. Taking the Fourier transform of the Langevin equation (5) and rearranging gives

$$
(-i \omega \mathbf{I}-\mathbf{A}) \tilde{\zeta}(\omega)=\tilde{\lambda}(\omega), \quad\langle\tilde{\lambda}(\omega) \tilde{\lambda}(-\omega)\rangle=T \mathbf{B} .
$$

Here $T$ is simply the duration of the simulation and we have used the approximation $\tilde{f}=-i \omega \tilde{f}$. This approximation is only accurate for systems tending to a stable, stationary steady state [12]. We can now calculate the power spectrum (the dagger denotes the complex transpose):

$$
\left\langle|\tilde{\zeta}(\omega)|^{2}\right\rangle=T(-i \omega \mathbf{I}-\mathbf{A})^{-1} \mathbf{B}(-i \omega \mathbf{I}-\mathbf{A})^{-1 \dagger} .
$$

\section{Spatiotemporal power spectrum}

To calculate the power spectrum in both time and space, we need to take the discrete cosine transform of the Langevin equation (5). We take the discrete cosine transform, rather than the discrete Fourier transform, as this automatically imposes the no-flux boundary conditions. For a function $f$ the discrete cosine transform, or discrete cosine series expansion, is defined as [12]

$$
\hat{f_{k}}=\Delta_{x} \sum_{j=1}^{K} \cos \left[k \Delta_{x}(j-1)\right] f\left(x_{j}\right) .
$$

Before applying this to the Langevin equation, let us note that we can write the matrices in (5) as $\mathbf{A}=\mathbf{A}_{0}+\mathbf{A}_{1}$ and $\mathbf{B}=\mathbf{B}_{0}+\mathbf{B}_{1}$, where $\mathbf{M}_{0}$ has only the diagonal entries of the submatrices and $\mathbf{M}_{1}$ only the sub- and superdiagonal ones, $\mathbf{M}$ being either $\mathbf{A}$ or $\mathbf{B}$. The discrete cosine transform of the Langevin equation (5) can then be written as

$$
\begin{aligned}
\frac{\mathrm{d} \hat{\zeta}_{k}(t)}{\mathrm{d} t} & =\left[\mathbf{A}_{0}+2 \cos \left(k \Delta_{x}\right) \mathbf{A}_{1}\right] \hat{\zeta}_{k}(t), \\
\left\langle\hat{\boldsymbol{\lambda}}_{k}(t) \hat{\boldsymbol{\lambda}}_{k}^{\dagger}\left(t^{\prime}\right)\right\rangle & =\frac{\Delta_{x}^{2} K}{2}\left[\mathbf{B}_{0}+2 \cos \left(k \Delta_{x}\right) \mathbf{B}_{1}\right] \delta\left(t-t^{\prime}\right) .
\end{aligned}
$$


From here we can proceed as in Sec. IIC3. Applying the temporal Fourier transform and rearranging, we have

$$
\tilde{\hat{\zeta}}_{k}(\omega)=\left[-i \omega \mathbf{I}-\mathbf{A}_{0}-2 \cos \left(k \Delta_{x}\right) \mathbf{A}_{1}\right]^{-1} \tilde{\hat{\lambda}}_{k}(\omega),
$$

which has reduced the number of coupled equations from $2 \mathrm{~K}$ to two and from which we finally obtain the full spatiotemporal power spectrum

$$
\mathbf{P}_{k}(\omega)=\left\langle\tilde{\hat{\zeta}}_{k}(\omega) \tilde{\hat{\zeta}}_{k}^{\dagger}(\omega)\right\rangle=T \boldsymbol{\Theta}^{-1} \boldsymbol{\Lambda} \boldsymbol{\Theta}^{-1 \dagger},
$$

where

$$
\boldsymbol{\Theta}=\left(\begin{array}{cc}
-i \omega-a_{0}-2 \cos \left(k \Delta_{x}\right) a_{1} & -b_{0} \\
-c_{0} & -i \omega-d_{0}-2 \cos \left(k \Delta_{x}\right) d_{1}
\end{array}\right),
$$

$$
\boldsymbol{\Lambda}=\frac{\Delta_{x}^{2} K}{2}\left(\begin{array}{cc}
\alpha_{0}+2 \cos \left(k \Delta_{x}\right) \alpha_{1} & \beta_{0} \\
\beta_{0} & \gamma_{0}+2 \cos \left(k \Delta_{x}\right) \gamma_{1}
\end{array}\right) .
$$

All components of the power spectrum can be straightforwardly evaluated, but for the purpose of this study we only need to consider the power spectrum of the first species

$$
\begin{aligned}
P[u, u]_{k}(\omega) & =\left\langle\tilde{\hat{\eta}}_{u k}(\omega) \tilde{\hat{\eta}}_{u k}^{\dagger}(\omega)\right\rangle \\
& =\frac{T \Delta_{x}^{2} K}{2} \frac{\alpha_{k}\left(\omega^{2}+d_{k}^{2}\right)}{\left(\omega^{2}+b_{0} c_{0}-a_{k} d_{k}\right)^{2}+\omega^{2}\left(a_{k}+d_{k}\right)^{2}},
\end{aligned}
$$

where we have introduced the shorthand $m_{k}=m_{0}+$ $2 \cos \left(k \Delta_{x}\right) m_{1}$, where $m$ is $a, d$, or $\alpha$.

\section{Stochastic simulations}

Simulations of the Schnakenberg system were carried out using the Gibson-Bruck algorithm [20] within the software package DIZZY [21], version 1.11.3. The parameters used are (unless otherwise stated) $\kappa_{1}=15, \kappa_{-1}=0.2, \kappa_{2}=43$, $\kappa_{3}=10^{-5}, D_{u}=10^{-4}, D_{v}=10^{-2}$, and domain length $L=$ 0.1 , discretized into $K=40$ compartments. Populations were initialized at steady state values throughout.

\section{E. Deterministic simulations}

Deterministic simulations were carried out in COMSOL MULTIPHYSICS 4.3. We chose initial conditions as a perturbation of Gaussian shape about the spatially uniform steady state. This was of magnitude $1 / 100$ relative to the steady state value, centered at $x=0$, and of width $\sigma=L / 4$, unless otherwise stated. We are aware that these perturbations may be seeding patterns with more power in the lower spatial modes, but since we are simply aiming to showcase possible dynamics of the system this should not be an issue.

\section{F. Conditions for stability, oscillations, and Turing instabilities}

Even though we identify spatial heterogeneity and oscillatory behavior in our simulations using power spectral methods, for comparison it is useful to recall the conditions derived from linear stability analysis in the deterministic system. Here it useful to nondimensionalize our equations first, as it reduces the number of free parameters. Our chosen nondimensionalization is

$$
\begin{aligned}
\frac{\partial \phi}{\partial t} & =\nabla^{2} \phi+\alpha-\phi+\phi^{2} \psi, \\
\frac{\partial \psi}{\partial t} & =D \nabla^{2} \psi+\beta-\phi^{2} \psi,
\end{aligned}
$$

where $\phi=\sqrt{c_{3} / c_{-1}} u, \psi=\sqrt{c_{3} / c_{-1}} v, D=D_{v} / D_{u}, t=$ $c_{-1} t^{\prime}$, and $x=\sqrt{c_{-1} / D_{u}} x^{\prime}$ if the primed variables are the dimensional ones. The nondimensional reaction rates are $\alpha=$ $c_{1} / c_{-1} \sqrt{c_{3} / c_{1}}$ and $\beta=c_{2} / c_{-1} \sqrt{c_{3} / c_{1}}$. The Jacobian of our deterministic, nondimensionalized reaction system is given by

$$
\mathbf{J}(\phi, \psi)=\left(\begin{array}{cc}
-1+2 \phi \psi & \phi^{2} \\
-2 \phi \psi & -\phi^{2}
\end{array}\right) .
$$

When evaluated at steady state we simply add the subscript $\mathbf{J}_{s}=\mathbf{J}\left(\phi_{s}, \psi_{s}\right)$, where $\phi_{s}=\alpha+\beta$ and $\psi_{s}=\beta / \phi_{s}^{2}$ is the steady state of the dimensionless Schnakenberg equations (17). From this, one can readily derive the stability eigenvalues, i.e., the growth rates of perturbations to the steady state,

$$
\lambda_{ \pm}(k)=\frac{\tau_{J}(k) \pm \sqrt{\tau_{J}(k)^{2}-4 \delta_{J}(k)}}{2} .
$$

We have introduced the shorthand notation, similarly to that in [22], $\tau_{J}(k)=\operatorname{tr}\left(\mathbf{J}_{s}-k^{2} \mathbf{D}\right)$ and $\delta_{J}(k)=\operatorname{det}\left(\mathbf{J}_{s}-k^{2} \mathbf{D}\right)$, where $k=m \pi / L$ denotes the wave number of the cosine expansion of the perturbation. Note that care has to be taken to use the dimensional or dimensionless forms of $k$ and $L$ where necessary.

Let us first consider the steady states in the absence of diffusion, which here is equivalent to setting $k=0$. From (19), the condition for linear stability of the Schnakenberg reaction system is

$$
\tau_{J}(0)=-\phi_{s}^{2}+2 \phi_{s} \psi_{s}-1<0 .
$$

Whether response to a perturbation is oscillatory or not is determined by the imaginary part of the eigenvalues of the stability matrix [5]. For the system to respond to perturbations in an oscillatory manner, stable or not, the eigenvalues need to be complex, which occurs for

$$
\tau_{J}(0)^{2}-4 \delta_{J}(0)<0,
$$

which can be solved to give

$$
\frac{\left(\phi_{s}-1\right)^{2}}{2 \phi_{s}}<\psi_{s}<\frac{\left(\phi_{s}+1\right)^{2}}{2 \phi_{s}} .
$$

The condition for Turing patterns is for an otherwise stable steady state to be destabilized through diffusion, which translates to

$$
\tau_{J}(0)<0, \quad \delta_{J}(k)<0
$$

or, explicitly,

$$
\psi_{0}>\frac{\left(\phi_{s}+\sqrt{D}\right)^{2}}{2 D \phi_{s}} .
$$

For a phase space diagram see Fig. 2. 


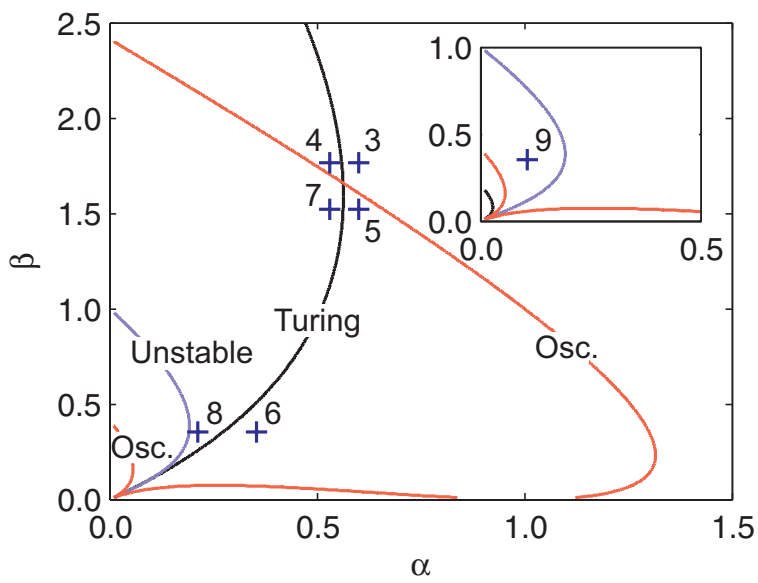

FIG. 2. (Color online) Parameter space of the deterministic Schnakenberg system in terms of the dimensionless reaction rates $\alpha$ and $\beta$. Turing instabilities occur to the left of the black line, given by (24). Oscillatory response to perturbations occurs between the red lines, given by (22). Steady states become unstable to the left of the blue line, given by (20). Sampled points are labeled with the corresponding figure number.

\section{Turing-unstable wave modes}

In addition to the conditions derived above, the domain on which the reactions take place has to be large enough for patterns to form. For a given perturbation, we can find which wave modes have a positive growth rate, i.e., are linearly unstable, by solving $\delta_{J}<0$ for $k$. For the dimensional Schnakenberg kinetics, we find the smallest Turing-unstable wave number $k_{T_{-}}$and the largest $k_{T_{+}}$to be given by

$k_{T \pm}^{2}=\frac{f_{u}}{2 D_{u}}+\frac{g_{v}}{2 D_{v}} \pm \sqrt{\left(\frac{f_{u}}{2 D_{u}}+\frac{g_{v}}{2 D_{v}}\right)^{2}-2\left(f_{v} g_{u}-f_{u} g_{v}\right)}$,

where $f_{u}, f_{v}, g_{u}$, and $g_{v}$ denote the partial derivatives of the reaction terms in the RDEs ( $f$ and $g$ for species $u$ and $v$, respectively) with respect to the subscript variable, i.e., the entries of the Jacobian (18), evaluated at steady state (explicitly, $f_{u}=-c_{-1}+2 c_{3} u_{s} v_{s}, f_{v}=c_{3} u_{s}^{2}, g_{u}=-2 c_{3} u_{s} v_{s}$, and $g_{v}=-c_{3} u_{s}^{2}$ ). We give the expression for the dimensional system to more easily compare with the length and time scales of our stochastic simulations, but the Turing-unstable wave modes $m=k L / \pi$ are the same as in the nondimensionalized case.

\section{G. Turing-Hopf instabilities}

Even though we are primarily interested in stochastic behavior, i.e., noise-induced spatiotemporal behavior, it will be useful to consider where behavior similar to polarity switching, namely, oscillating or twinkling patterns [22], is predicted deterministically. Oscillating patterns are said to occur through a strong Turing-Hopf instability [22], for which the steady state needs to be unstable and perturbations lead to oscillatory behavior. Therefore, Turing-Hopf instabilities require complex stability eigenvalues with a positive real part. Furthermore, this has to occur for particular wave numbers, i.e.,

$$
\tau_{J}(k)>0, \quad \tau_{J}(k)^{2}-4 \delta_{J}(k)<0,
$$

and both conditions have to hold for the same $k>0$ (although multiple $k$ can be unstable and have complex stability eigenvalues at the same time). For $k=0$ we reproduce the conditions for the background to become unstable and oscillatory.

\section{Oscillating wave modes}

Solving (26) for $k$, we obtain an expression similar to (25), only now it predicts which wave modes contribute to the deterministically predicted oscillating pattern rather than the static Turing pattern. The stability eigenvalues become unstable for wave numbers

$$
k^{2}<\frac{f_{u}+g_{v}}{D_{u}+D_{v}},
$$

and the highest and lowest wave numbers for which the stability eigenvalues become complex are

$$
k_{\mathbb{C} \pm}^{2}=\frac{f_{u}-g_{v} \pm 2 \sqrt{-f_{v} g_{u}}}{D_{u}-D_{v}},
$$

with $f_{u}, f_{v}, g_{u}$, and $g_{v}$ as in (25) and $D_{u} \neq D_{v}$.

\section{Dispersion relation}

Wave modes with complex stability eigenvalues will oscillate with frequency [22]

$$
\omega(k)=\sqrt{\delta_{J}-\tau_{J}^{2} / 4} .
$$

For the dimensional system, this can be written as

$$
\omega(k)=\sqrt{\omega_{0}^{2}+\frac{\left(f_{u}-g_{v}\right)\left(D_{u}-D_{v}\right) k^{2}}{2}-\frac{\left(D_{u}-D_{v}\right)^{2} k^{4}}{4}},
$$

where $\omega_{0}^{2}=-g_{u} f_{v}-\left(f_{u}-g_{v}\right)^{2} / 4$ is the squared frequency of background oscillations, i.e., for wave mode $m=0$. From this we can distinguish between the cases $D_{u}>D_{v}$ and $D_{u}<$ $D_{v}$ with respect to the shape of the dispersion relation. If $f_{u}-g_{v}>0(<0)$, then we can only have patterns oscillating at higher frequencies than the background for $D_{u}<D_{v}\left(>D_{v}\right)$ and the dispersion relation is maximized for $k_{\omega_{\max }}^{2}=\left(f_{\phi}-\right.$ $\left.g_{\psi}\right) /(1-D)$.

\section{RESULTS}

Note that when showing the results of simulations, we have limited the range of values displayed for visual emphasis of patterns, oscillations, or lack thereof. The results of deterministic simulations have been scaled by the system size to ease visual comparison with the stochastic simulation results.

\section{A. Outside the oscillatory and Turing regimes}

Near the boundaries for the onset of Turing patterns and oscillatory response to perturbations, we find stochastic patterns, but no background oscillations (Fig. 3). The power spectra of stochastic simulations only have appreciable power in the first nonzero spatial mode, but not the zeroth. There 


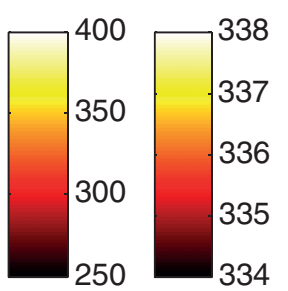

(b) stochastic
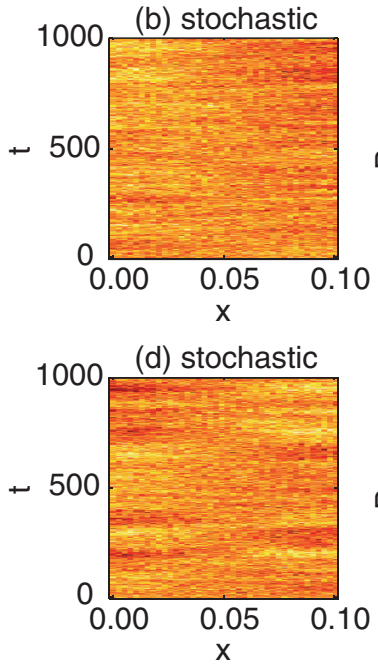

(f) analytical

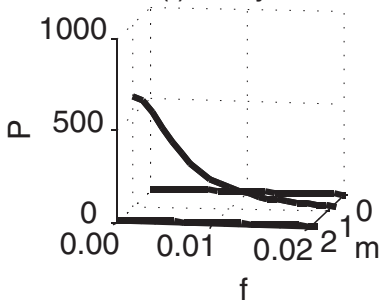

(a) deterministic

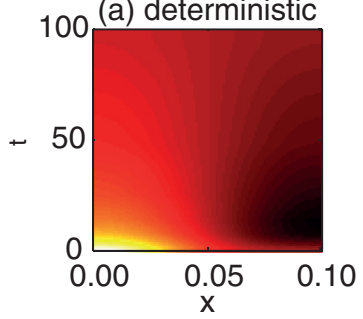

(c) spectrum of (b)

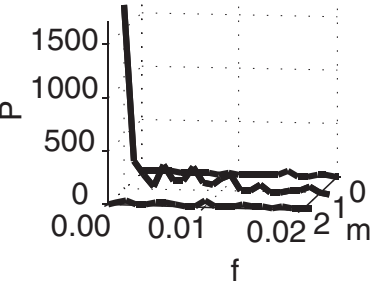

(e) spectrum of (d)

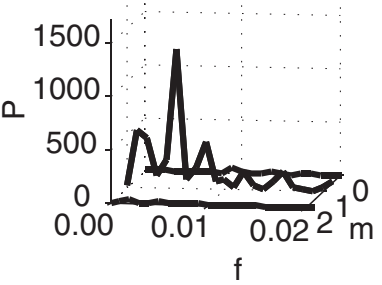

(g) average

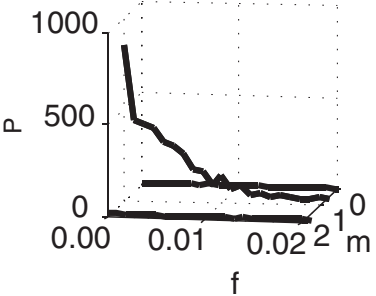

FIG. 3. (Color online) Just outside the Turing and oscillatory regimes, (a) the deterministic simulation shows a rapid decay from the initial perturbation. The stochastic simulations show both (b) and (c) static patternlike behavior and (d) and (e) oscillatory patterns in individual realizations. (g) On average, the stochastic behavior is patternlike without a defined frequency of oscillations (40 realizations), as predicted by (f) the analytic spectrum of the Langevin dynamics. The parameters used are $\kappa_{1}=17, \kappa_{2}=50$, and the rest are as given in Sec. IID. The right color bar corresponds to the deterministic results, while the left color bar corresponds to the stochastic results.

are also realizations for which the power in the first spatial mode is peaked at a nonzero frequency, a sign of (stochastic) oscillatory patterns. However, these do not consistently occur at a defined frequency across realizations.

\section{B. Inside the Turing regime, outside the oscillatory regime}

Crossing into the Turing regime, but staying outside the region predicted to produce oscillatory response to perturbations, the stochastic simulations produce power spectra with pronounced peaks in the first spatial mode at or near zero frequency (Fig. 4). Thus we again see patterns and oscillatory patterns, much like in Sec. III A. Even though we are inside the
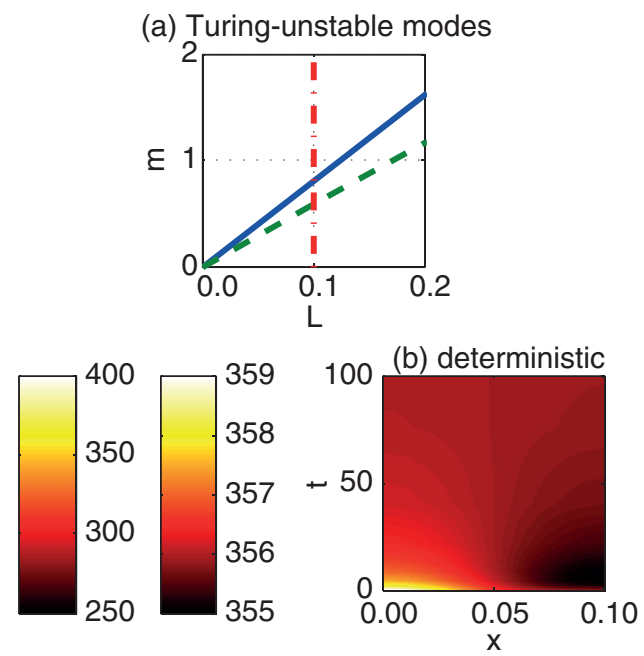
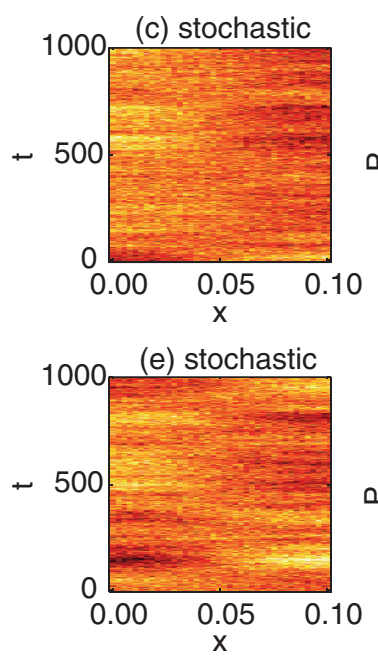

(g) analytical

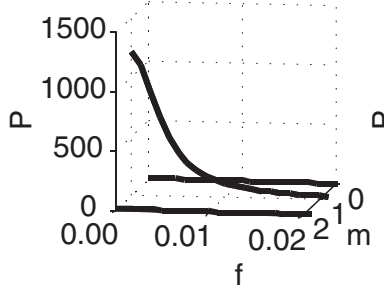

(d) spectrum of (c)

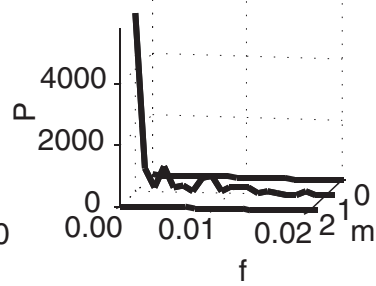

(f) spectrum of (e)

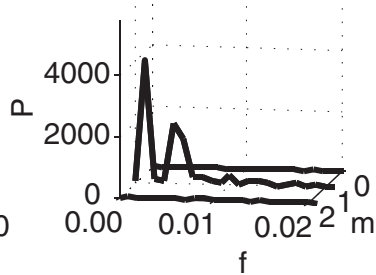

(h) average

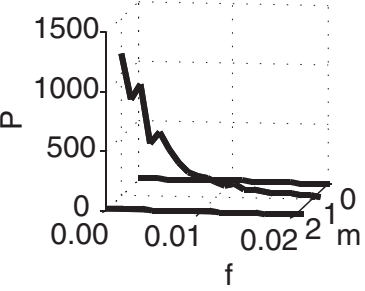

FIG. 4. (Color online) Just inside the Turing regime, just outside the oscillatory regime, but (a) without Turing-unstable wave modes, (b) the deterministic simulation shows a rapid decay back to the spatially uniform steady state from the initial perturbation. Shown in (a) are the highest Turing-unstable wave mode (blue solid line) and lowest (green dashed line) as given by (25). The red dash-dotted line shows the domain length used. The stochastic simulations show both (c) and (d) static patternlike behavior and (e) and (f) oscillatory patterns in individual realizations. (h) On average, the stochastic behavior is patternlike without a defined frequency of oscillations (40 realizations), as predicted by $(\mathrm{g})$ the analytic spectrum of the Langevin dynamics. The parameters used are $\kappa_{2}=50$ and the rest are as in Sec. IID. The right color bar corresponds to the deterministic results, while the left color bar corresponds to the stochastic results.

region where Turing instabilities form, we intentionally keep the system too small for wave modes to become Turing 

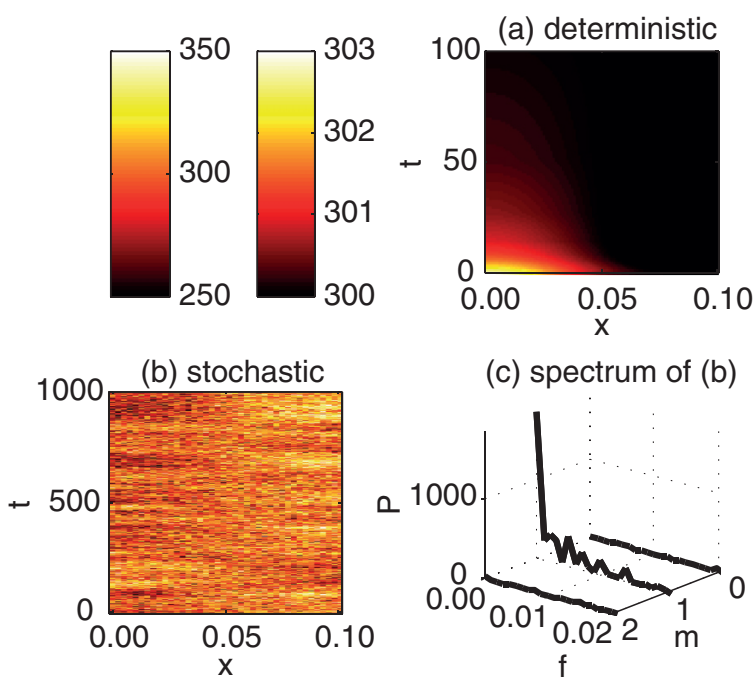

(c) spectrum of (b)

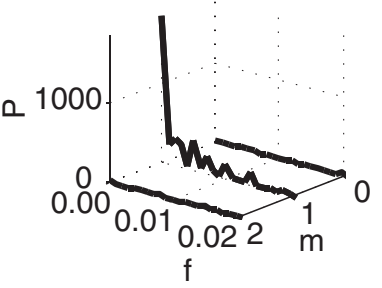

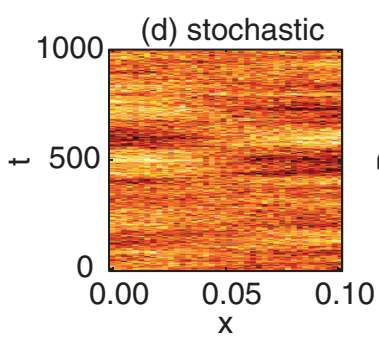

(f) analytical

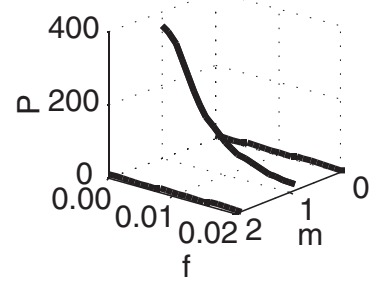

(e) spectrum of $(d)$

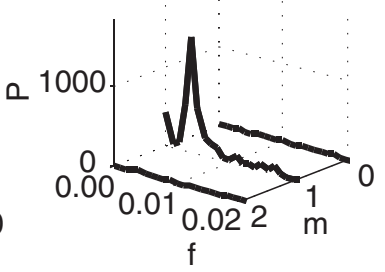

(g) average

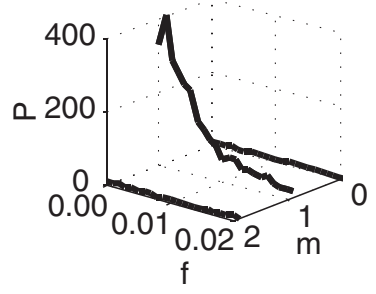

FIG. 5. (Color online) Just outside the Turing regime, just inside the oscillatory regime, (a) the deterministic simulation shows a rapidly decaying perturbation. Additionally, perturbations of higher magnitude (1/10 of the steady state value) did not produce visible oscillations. The stochastic simulations show both (b) and (c) static patternlike behavior and (d) and (e) oscillatory patterns in individual realizations. (g) On average, the stochastic behavior is patternlike without a defined frequency of oscillations (40 realizations), as largely predicted by (f) the analytic spectrum of the Langevin dynamics. The parameters used are $\kappa_{1}=17$ and the rest as in Sec. IID. The right color bar corresponds to the deterministic results, while the left color bar corresponds to the stochastic results.

unstable. Hence we do not expect patterns to form in the deterministic simulation.

\section{Outside the Turing regime, inside the oscillatory regime}

Crossing into the oscillatory instead of the Turing regime, the behavior initially does not change much: The power spectra of individual realizations show signs of stochastic patterns and oscillatory patterns, but not background oscillations (Fig. 5), which are what we would expect deterministically. However, a deterministic simulation bears no obvious signs of oscillations, at least not at the chosen duration and temporal resolution.

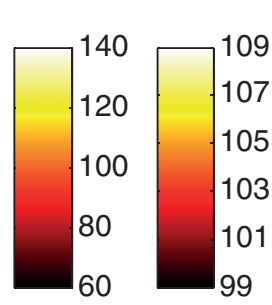

(b) stochastic

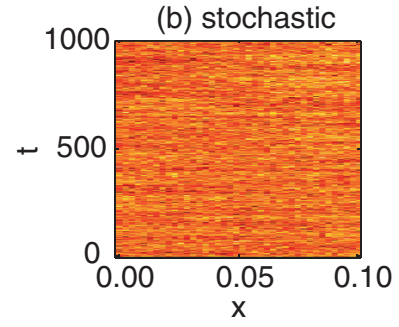

(d) stochastic

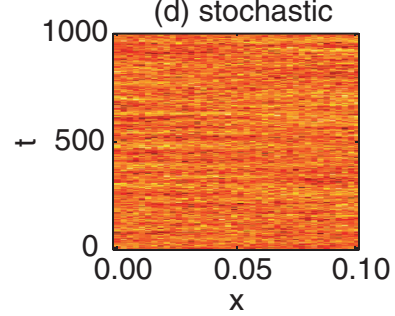

(f) analytical

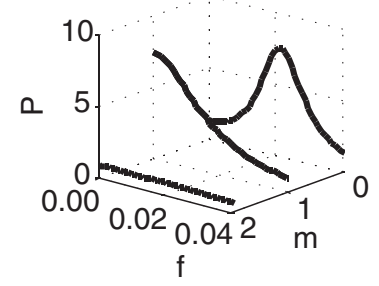

(a) deterministic

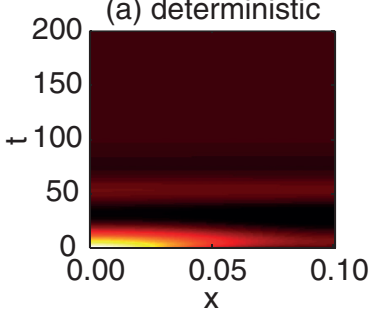

(c) spectrum of (b)

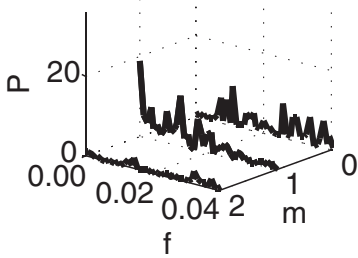

(e) spectrum of (d)

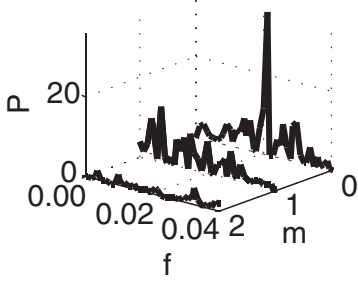

(g) average

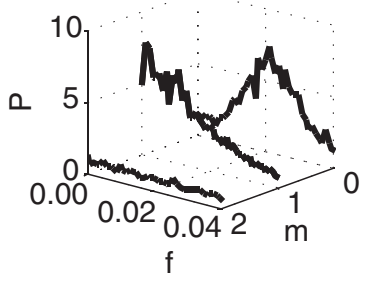

FIG. 6. (Color online) Just outside the Turing regime, far inside the oscillatory regime, (a) the deterministic simulation shows an oscillatory decay of the perturbation. Note that the perturbation here is larger than usual; its magnitude is $1 / 10$ of the steady state value. (b) and (d) The stochastic simulations are difficult to classify, but the power spectra have peaks characteristic of both (c) patterns and (d) background oscillations, if at very low power. (g) On average, the stochastic behavior is patternlike with background oscillations around a defined frequency (40 realizations), as predicted by (f) the analytic spectrum of the Langevin dynamics. The parameters used are $\kappa_{1}=10, \kappa_{2}=10$, and the rest as in Sec. II D. The right color bar corresponds to the deterministic results, while the left color bar corresponds to the stochastic results.

Background oscillations become more pronounced in both the deterministic and stochastic simulations when we move further into the oscillatory regime and closer to the region of (linearly) unstable steady states (Fig. 6). Individual power spectra are of very low magnitude and noisy, but the average agrees well with the analytical spectrum.

\section{Inside the Turing and oscillatory regimes}

Crossing into both the Turing and oscillatory regimes, while staying near the boundaries of both, the system once again produces stochastic patterns and oscillatory patterns, but 
(a) Turing-unstable modes

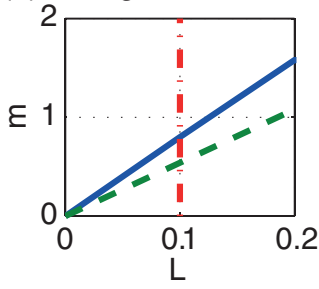

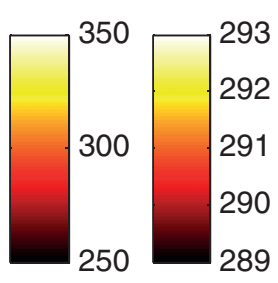

(c) stochastic

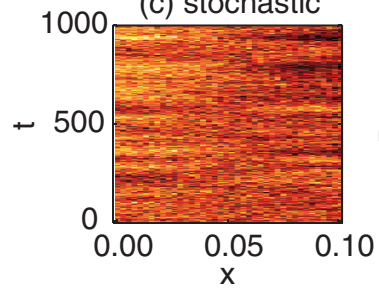

(e) stochastic

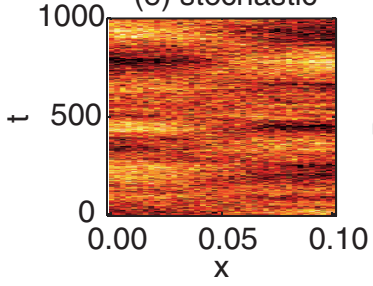

(g) analytical
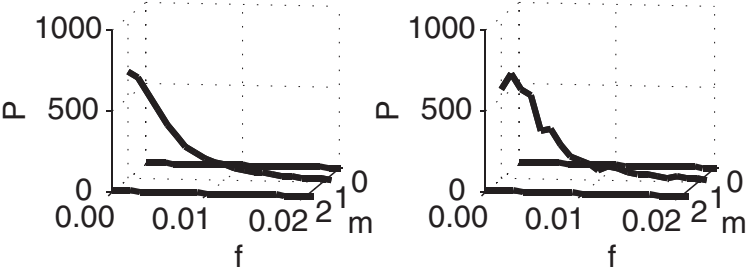

FIG. 7. (Color online) Just inside the Turing regime and just inside the oscillatory regime, but (a) without Turing-unstable wave modes, (b) the deterministic simulation shows a rapidly decaying perturbation. Perturbations of higher magnitude (1/10 of the steady state value) did not produce visible oscillations. The stochastic simulations show both (c) and (d) static patternlike behavior and (e) and (f) oscillatory patterns in individual realizations. (h) On average, the stochastic behavior is patternlike without a defined frequency of oscillations (40 realizations), as predicted by (g) the analytic spectrum of the Langevin dynamics. The parameters used are as in Sec. II D. The right color bar corresponds to the deterministic results, while the left color bar corresponds to the stochastic results. In (a) the highest Turing-unstable wave mode (blue solid line) and lowest (green dashed line) are as given by (25). The red dash-dotted line shows the domain length used.

again not at a consistently defined frequency (Fig. 7). Moving closer towards the predicted loss of stability, background oscillations become more pronounced (Fig. 8). In individual realizations they can carry more power than the patterning, (a) Turing-unstable modes
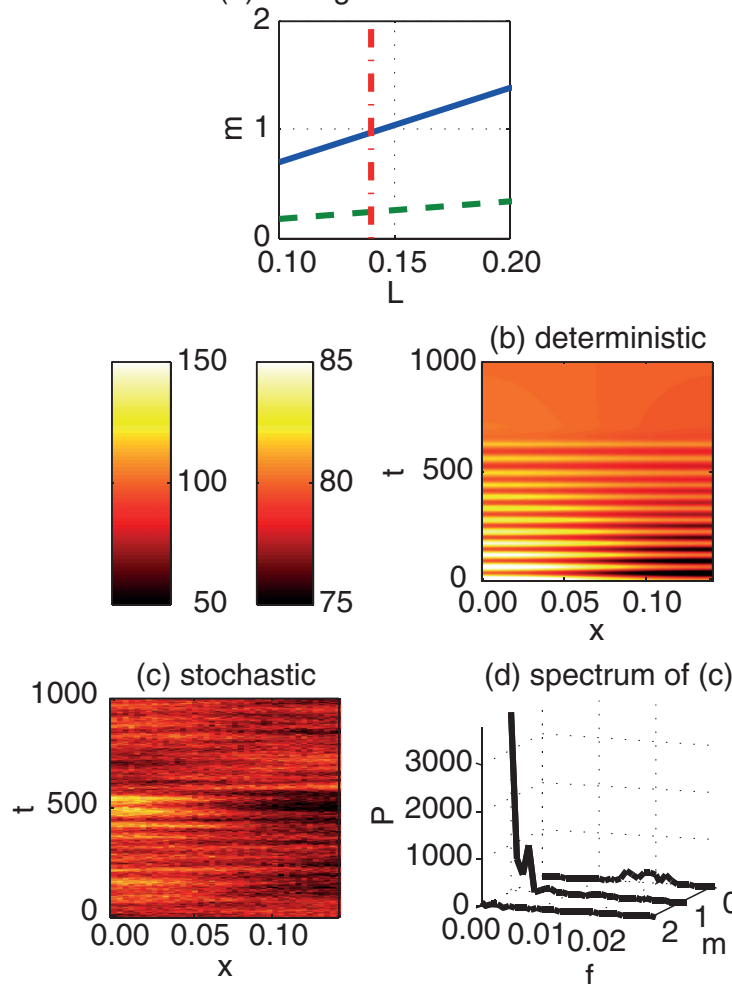

(d) spectrum of (c)

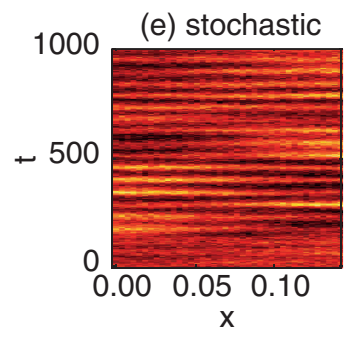

(g) analytical
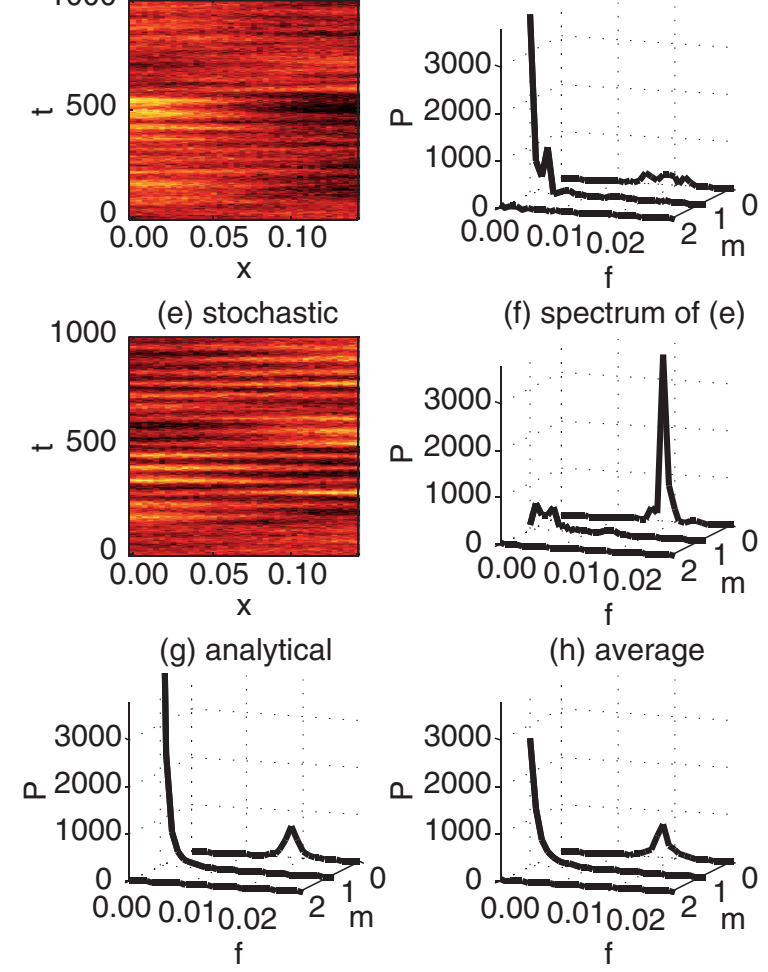

(f) spectrum of (e)

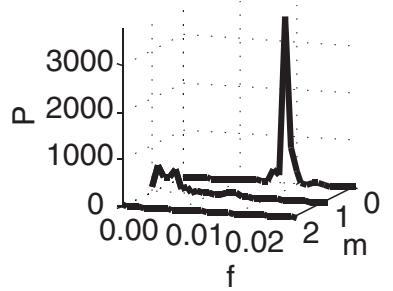

(h) average

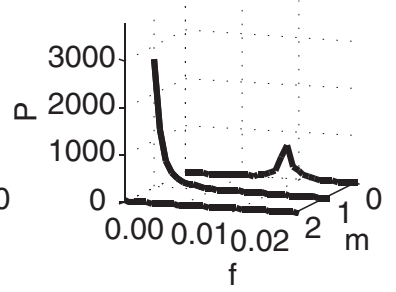

FIG. 8. (Color online) Just inside the Turing regime, within the oscillatory regime and just outside the unstable regime, but (a) without Turing-unstable wave modes, (b) the deterministic simulation shows an oscillatory decay of the perturbation. Note that the perturbation here is larger than usual; its magnitude is $1 / 10$ of the steady state value. The stochastic simulations show both (c) and (d) static patternlike behavior and (e) and (f) background oscillations in individual realizations. Most realizations showcase both, as in (h) the average spectrum (29 realizations). (g) The analytic spectrum of the Langevin dynamics predicts the background oscillations as well as the patterning, but the latter not at the right magnitude. The parameters used are $\kappa_{1}=6, \kappa_{2}=10, L=0.14$, and the rest as in Sec. IID. The right color bar corresponds to the deterministic results, while the left color bar corresponds to the stochastic results. In (a) the highest Turing-unstable wave mode (blue solid line) and lowest (green dashed line) are as given by (25). The red dash-dotted line shows the domain length used. 
but on average most of the power stays concentrated in the first spatial mode. Compared to Sec. III C, Fig. 6, the peak in the zeroth mode, corresponding to background oscillations, is more pronounced, but at comparatively lower power with respect to the first mode. This point in parameter space also gives us the most sustained oscillations in the deterministic simulation out of our samples.

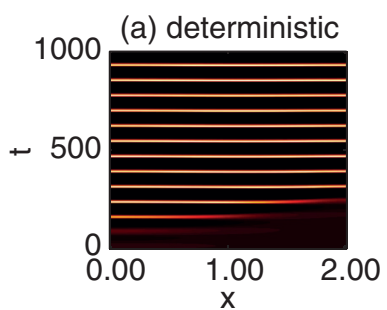

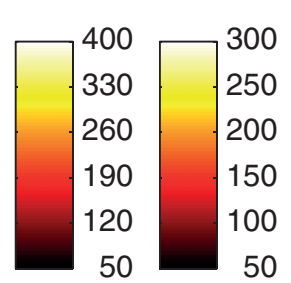

(c) stochastic

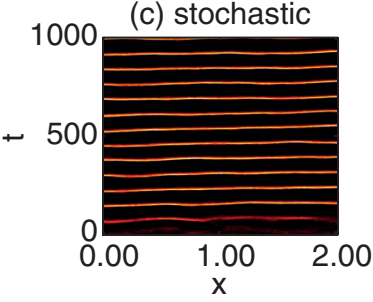

(e) stochastic

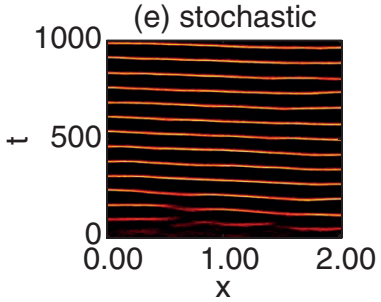

(g) analytical

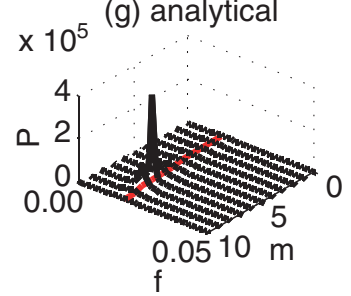

(b) close-up of $A$

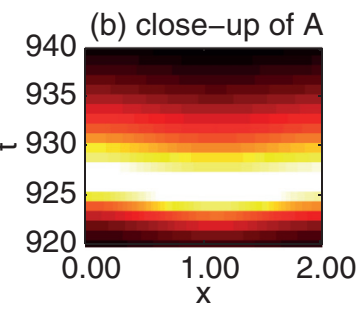

(d) spectrum of (c)

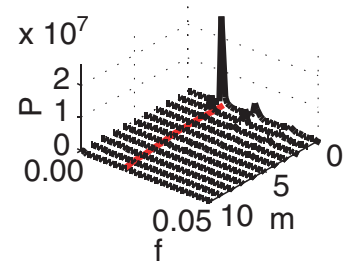

(f) spectrum of (e)

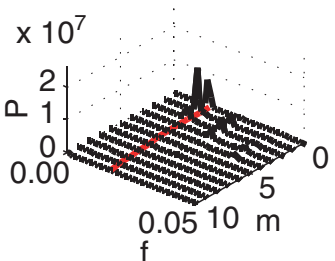

(h) average

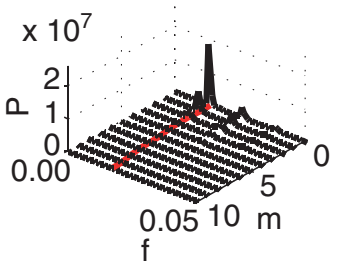

FIG. 9. (Color online) Far from the Turing regime, inside the oscillatory and unstable regimes, (a) and (b) the deterministic simulation shows fast-traveling waves. (c) and (e) The stochastic simulations also show waves, but visibly slower. (d) and (f) The sharp wave peaks are constructed of multiples of frequencies, which is (h) consistent across realizations ( 10 realizations). As the assumptions underlying the Langevin dynamics break down, (g) the analytic spectrum fails to predict the observed shape. The red dashed line shows the dispersion relation. The parameters used are $\kappa_{1}=3$, $\kappa_{2}=10, D_{u}=4 \times 10^{-4}, D_{v}=10^{-4}, L=2, K=80$, and the rest as in Sec. IID. The right color bar corresponds to the deterministic results, while the left color bar corresponds to the stochastic results.

\section{E. Inside the strong Turing-Hopf regime}

Finally, we consider a region of parameter space where strong Turing-Hopf bifurcations are predicted to occur deterministically. We find traveling waves in both the deterministic simulation and the stochastic realisations (Fig. 9). However, the waves in the stochastics travel at a slower speed, which can be seen as they form an angle further from the horizontal in the space-time plot. The stochastic traveling waves also display more heterogeneity between individual oscillations. Since we are in the region of unstable steady states, the analytic spectrum derived from the Langevin dynamics, which assume a stable steady state, now fails to approximate the average observed power spectrum.

\section{DISCUSSION}

We set out to characterize the role of intrinsic noise in pattern formation, in particular with regard to spatiotemporal dynamic behavior. Using stochastic simulations, we explored the parameter space of the Schnakenberg system and compared our findings against analytical results that we derived from the master equation under a weak-noise approximation.

\section{A. Polarity switching}

Through power spectral methods, we were able to identify cases of oscillatory polarity switching in a range of places in the parameter space of the Schnakenberg reaction system. The signature we were looking for are power spectral peaks at nonzero frequencies and nonzero spatial modes. These are most pronounced near and inside the region of Turing instabilities (Figs. 3-5 and 7). The frequencies of oscillation can be pronounced and narrowly defined in individual realisations of the stochastic simulations, but are not consistently so across realizations, as they do not appear in the averaged power spectra. This is in agreement with analytical predictions derived from the Langevin equation, which is an average over an infinite ensemble of theoretical realisations. Indeed, closer inspection of (27) reveals that linear stability analysis is unable to predict oscillating Turing patterns (and instead only Turing patterns with background oscillations), as the eigenvalues of Turing-unstable modes are always real.

The analytic results are successful at predicting coexisting patterns and background oscillations (Figs. 6 and 8). Similar power spectra with peaks at $k=0, f>0$ and $f=0, k>0$ have been reported in predator-prey systems [5].

\section{B. Traveling waves}

By turning to Turing-Hopf analysis we also investigated where oscillating patterns, or traveling waves, would be expected deterministically. Where the deterministic system undergoes a strong Turing-Hopf bifurcation (26), the stochastic system exhibits similar behavior. However, in most of the stochastic realisations the traveling wave speed is visibly slower than in the deterministic simulation (Fig. 9). There is some variability between stochastic realizations, but the underlying deterministic behavior dominates. Deterministically, the traveling waves are dependent on the initial conditions, so the comparison is only qualitative. The Langevin-based analytics 
fail to predict the average power spectrum of the stochastic simulations since the assumptions under which they were derived do not hold true when the steady states are unstable.

Purely noise-induced traveling waves have been reported by Biancalana et al. [10] in the Brusselator system, which is similarly canonical in pattern formation to the Schnakenberg system. These were much lower in amplitude and required an additional nonlocal interaction. It remains to be seen whether suitably strong, visibly oscillating patterns or traveling waves can be purely noise induced on top of a deterministically stable, homogeneous steady state in a general reaction system without nonlocal modifications. Like in the case of stochastic pattern formation, intrinsic noise may widen the parameter region for Turing-Hopf bifurcations, but to find a clear case of this likely amounts to a parameter fine-tuning problem in itself.

\section{Conclusions}

Based on the wide but necessarily limited range of parameters sampled, we conclude that intrinsic noise cannot consistently induce a specific frequency of polarity switching in stochastic Turing patterns. This is unlike the ability of noise to induce patterns and background oscillations. However, intrinsic noise can have a consistent, visible effect on the spatiotemporal dynamics predicted under a Turing-Hopf bifurcation. This cannot be captured by the usual Langevin approach, but one might be able to investigate further by considering fluctuations on top of an oscillatory steady state. We have only investigated the Schnakenberg kinetics in one spatial dimension, but expect similar results in other reaction systems, and it may be interesting to study noise-induced polarity switching in higher spatial dimensions.

\section{ACKNOWLEDGMENTS}

We gratefully acknowledge the U.K.'s Engineering and Physical Sciences Research Council (EPSRC) for funding through a studentship (L.J.S.) at the Life Science Interface programme of the University of Oxford's Doctoral Training Centre. This publication is based on work supported by Award No. KUK-C1-013-04, made by King Abdullah University of Science and Technology (KAUST).
[1] P. K. Maini, T. E. Woolley, R. E. Baker, E. A. Gaffney, and S. S. Lee, Interface Focus 2, 487 (2012).

[2] A. M. Turing, Phil. Trans. R. Soc. Lond. B 237, 37 (1952).

[3] T. Butler and N. Goldenfeld, Phys. Rev. E 80, 030902 (2009).

[4] T. Butler and N. Goldenfeld, Phys. Rev. E 84, 011112 (2011).

[5] C. A. Lugo and A. J. McKane, Phys. Rev. E 78, 051911 (2008).

[6] A. J. McKane and T. J. Newman, Phys. Rev. E 70, 041902 (2004).

[7] J. L. Aragón, R. A. Barrio, T. E. Woolley, R. E. Baker, and P. K. Maini, Phys. Rev. E 86, 026201 (2012).

[8] A. J. McKane, J. D. Nagy, T. J. Newman, and M. O. Stefanini, J. Stat. Phys. 128, 165 (2007).

[9] T. Dauxois, F. Di Patti, D. Fanelli, and A. J. McKane, Phys. Rev. E 79, 036112 (2009).

[10] T. Biancalani, T. Galla, and A. J. McKane, Phys. Rev. E 84, 026201 (2011).

[11] T. Biancalani, T. Rogers, and A. J. McKane, Phys. Rev. E 86, 010106 (2012).
[12] T. E. Woolley, R. E. Baker, E. A. Gaffney, and P. K. Maini, Phys. Rev. E 84, 021915 (2011).

[13] F. Zheng-Ping, X. Xin-Hang, W. Hong-Li, and O. Qi, Chin. Phys. Lett. 25, 1220 (2008).

[14] N. G. van Kampen, Stochastic Processes in Physics and Chemistry (Elsevier, Amsterdam, 1992).

[15] J. Elf and M. Ehrenberg, Genome Res. 13, 2475 (2003).

[16] A. Gierer and H. Meinhardt, Biol. Cybern. 12, 30 (1972).

[17] J. Schnakenberg, J. Theor. Biol. 81, 389 (1979).

[18] T. E. Woolley, R. E. Baker, E. A. Gaffney, and P. K. Maini, Phys. Rev. E 84, 041905 (2011).

[19] D. T. Gillespie, J. Chem. Phys. 113, 297 (2000).

[20] M. A. Gibson and J. Bruck, J. Phys. Chem. A 104, 1876 (2000).

[21] S. Ramsey, D. Orrell, and H. Bolouri, J. Bioinf. Comput. Biol. 3, 415 (2005).

[22] M. R. Ricard and S. Mischler, J. Nonlin. Sci. 19, 467 (2009). 